\title{
Multi-Robot Cooperation Strategy Based on Wireless Sensor Network
}

\author{
https://doi.org/10.3991/ijoe.v14i11.9514 \\ Bo Sun $\left({ }^{\varpi}\right)$, Jing-wei Li \\ Henan Institute of Technology. Xinxiang, Henan, China \\ bosun29103@163.com \\ Guan-ci Yang \\ Guizhou University, Guiyang, Guizhou, China
}

\begin{abstract}
To realize the design of multi-robot cooperative control based on wireless sensor networks, a large number of local control research experiments are carried out on a specific model of mobile robots. Combined with the kinematic model of mobile robot, the motion characteristics of this kind of mobile robot are grasped. On the basis of realizing the multi-target tracking and positioning of wireless sensor network, a multi mobile robot cooperative control system based on the feedback of wireless sensor network is developed, and the synchronous motion between the master and the robot is maintained through the feedback control of the network. Finally, a cooperative trajectory tracking control algorithm for mobile robot is introduced, and the principle of the controller design is mastered. A multi mobile robot cooperative trajectory tracking LQG controller is designed for mobile robot in wireless sensor network. The simulation results show that the algorithm can reach the cooperative control target.
\end{abstract}

Key Words—wireless sensor networks, multiple mobile robots, cooperative control

\section{$1 \quad$ Introduction}

Multi-mobile robot systems and networked remote control are hot research issues in the field of mobile robots. The control problem of mobile robot can be attributed to two types of stabilization and tracking, and positioning and navigation are the basis of control. Positioning and navigation through the sensor network. Networked remote control was implemented and positioning navigation problems were solved. The combination of mobile robots and wireless sensor networks is the inevitable result of the development of the discipline.

In the research of wireless sensor networks, mobile robots usually only serve as positioning targets. It is equipped with various types of sensors and can independently move according to local planning paths. There is no information feedback between the two. The research focuses on the wireless sensor networking mode, topology control, routing protocols, energy consumption and other aspects. 
However, the mobile robot can serve as a tracking target and as a control object in the network. A networked multi-mobile robot system was established. Its collaborative control has significant research significance.

Under the support of the National Natural Science Foundation of China, Prof. Su Weizhou of the School of Automation Science and Engineering of South China University of Technology led the core team to conduct research on cooperative control of multi-mobile robots based on wireless sensor network feedback. It is divided into two parts: wireless sensor network multi-target tracking system and networked multi-mobile robot control. The two are closely linked. In the networked multi-mobile machine cooperative control system, the mobile robot acquires the positioning and navigation data of the wireless sensor network and accepts motion commands. Based on this, it serves as an input to the controller. This achieves true network feedback control and can keep pace with other robots. The realization and stable operation of the platform means that multi-target real-time tracking and multi-mobile robot network feedback control technology has reached the leading level in the domestic counterparts.

Mobile robots and wireless sensor networks are currently hot topics in academia. The combination of the two forms an innovative network control system platform integrating sensor measurement, network feedback, and multi-agent control. As a physical experiment platform of the National Natural Science Foundation of China's key project "Network System Control Theory and Method $(60,774,057)$ ", this platform has designed and implemented a multi-mobile robot real-time tracking and feedback cooperative control system based on wireless sensor networks. Through the wireless sensor network, the mobile robot is positioned. After the robot acquires the positioning information, it moves according to the mission planning. The master and slave robots keep synchronized.

Based on the above background, the wireless sensor network and the multi-mobile robot are mainly combined, and the multi-robot cooperative strategy based on the wireless sensor network is mainly studied.

\section{State of the art}

Wireless sensor network (WSN) is a research hotspot in the 21 st century. It is a product of cross-disciplinary combination of computer, communication, control, and sensors, and it is also a complex measurement and control system integrating information acquisition, transmission, and processing. Palanikumar and Ramasamy [1] pointed out that wireless sensor networks consist of a large number of distributed sensor network nodes. Each node has limited computing processing, storage, and wireless communication capabilities to sense the surrounding environment. Korol et al. [2] pointed out that nodes and nodes communicate information through wireless communication and form an ad hoc network. Through the switchboard control, sensor data and information fusion results are passed to users.

Wireless sensor networks have received a lot of attention in academia. In practice, more and more practical application systems have emerged and brought convenience to human life. Tang et al. [3] summarized the typical WSN applications in recent years. 
Szczepny et al. [4] used WSN technology to implement intelligent parking management system. It can instantly transmit the latest parking information to vehicles in the parking lot so that the owner can search for parking spaces in time. Another example is the closed warehouse cargo management system, which witnessed the mature application of WSN technology in the field of logistics. In 2002, Intel Corporation established the world's first wireless vineyard in Oregon. WSN technology is used in agricultural applications. The wireless vineyard integrates precision and intelligent farming, which is of great significance for improving the intelligence of modern agriculture.

At present, the main research problems of network control systems are delay, packet loss, timing disorder, single-packet and multi-packet transmission. The main research methods are model control method, predictive control method, random control method, and handover control method. Zhu et al. [5] studied the Markov delay network control system, which is quite extensive. Oliveira et al. [6] proposed $\mathrm{H} 2 / \mathrm{H}$ control, which is widely used in NCS. Network control has become a cross-cutting research area for control systems, communications networks, computer science, and information theory.

Network control systems have a wide range of applications. The networked robot control system is one of the most active research contents. Berghuis et al. [7] pointed out that the networked robot control was originally accompanied by the application of network technology and developed from teleoperation robots. Teleoperation robot system consists of operators, robots, and communication networks. It is a human-computer interaction technology proposed in the 1980s. Samejima et al. [8] pointed out that remote-controlled operators are mainly responsible for system monitoring in the remote monitoring room. Abnormal conditions in the work environment are handled. Remote technology improves the flexibility of robot and equipment motion control, avoiding system instability and other issues.

In summary, the above research is mainly focused on the independent research of wireless sensor networks and multiple mobile robots, and lacks the research to fuse the two. Therefore, based on the above research status, a multi-robot cooperative strategy based on wireless sensor networks is studied. First, wireless sensor networks and multiple mobile robots are introduced. Then, wireless sensor networks are applied to multimobile robots to achieve multi-robot coordination.

\section{$3 \quad$ Methodology}

\subsection{An overview of the system}

The entire network feedback control system is mainly composed of ultrasonic sensor nodes, wireless communication modules, base stations, wheeled mobile robots, and upper computers. The platform is a distributed wireless sensor network platform.

The wireless sensor network locates and tracks the wheeled mobile robot and reports its coordinate information to the robot's local control terminal. The specific implementation process is as follows: First, the wireless sensor network is initialized, the humancomputer interaction interface is opened, and a start command is clicked. The working base station transmits the work node's work token and communication token to each 
node of the network through the ZigBee network. Second, using the wheeled robot of Shenzhen Ou Peng as the tracking target, it is put into the wireless sensor network. Third, the task node that gets the work token inspires Sonar to perform the ranging. Fourth, after the ranging is completed, the task node transmits the asynchronous measurement data to the monitoring base station through the ZigBee network in the order of the communication token. Fifth, after the monitoring base station performs data fusion on the asynchronous measurement data, the target position is calculated, and the target trajectory is displayed in real time through labview. Sixth, according to the latest position of the target, the base station generates a new work token and a communication token for the task node, and broadcasts it through ZigBee.

The experimental platform is unified by LabVIEW by monitoring the base station. It mainly includes data communication module, data management module, data analysis module, sensor data display module, real-time tracking display module and filtering module. LabVIEW management platform module of WSN tracking and positioning system is shown in Figure 1.

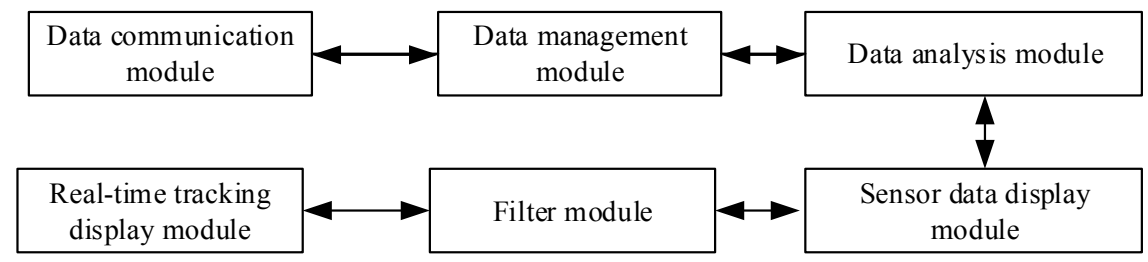

Fig. 1. LabVIEW management platform module of WSN tracking and positioning system

Under the role of the overall scheduling strategy and various functional modules, the wireless sensor network node can perform ultrasonic ranging on the moving target in the network and send the measured distance to the base station. LabVIEW obtains the position information of the target through the data fusion after the base station obtains the data, thereby realizing the positioning. The specific signal flow chart is as shown in Figure 2 .

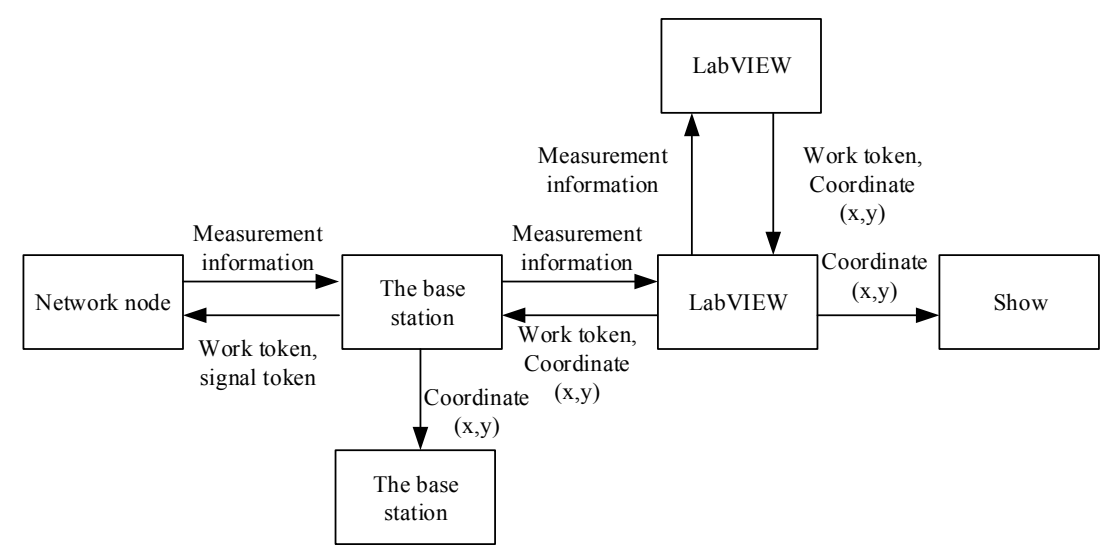

Fig. 2. Signal flow chart of WSN control system 


\subsection{Wireless sensor network multi-target tracking and positioning strategy}

There are several algorithms for wireless network sensor target positioning, such as least square method, Taylor-based incremental optimization algorithm and Kalman filter algorithm. After experimental comparison, the Kalman filtering algorithm works well and the generated trajectory is the smoothest. The second is an incremental optimization algorithm based on Taylor's expansion. The effect of only the least square method is not ideal. Therefore, the Kalman filter algorithm is used for positioning data processing in this project.

In a network, the multi-target tracking problem is much more complex than the single target. This network platform has the characteristics of distributed, high-speed asynchronous measurement data fusion, and uses ultrasonic measurement to simulate the physical model of a high-noise environment. The laboratory members of wireless sensor network positioning proposed a sensor task node scheduling strategy and node adaptive clustering and network routing protocol for inter-cluster negotiation. The previous single target tracking work mode was changed. The multi-target positioning and tracking in the network is realized, and a real-time feedback system is formed with the mobile robot, which can realize the precise navigation of the robot and the multi-robot formation control. It is highly innovative. Task scheduling strategies, mutual interference in ultrasonic ranging, communication channel bandwidth limitations, and distributed asynchronous measurement data fusion algorithms are key technical issues in the entire study. After many experiments, the platform can track the target's maximum speed to $1 \mathrm{rad} / \mathrm{s}$ (centered on the mission node).

\subsection{Data measurement and data fusion}

A large number of distributed sensors constitute a wireless sensor network. Nodes assigned to work tokens measure the distance to the target and summarize it to the LabVIEW master management platform. LabVIEW calls the corresponding function module for filtering and fusion calculation to get the global coordinate information of the mobile robot.

The target's motion model in the network is:

$$
X(k+1)=A(k) X(k)+w\left(k, \Delta \boldsymbol{t}_{k}\right)
$$

The target's status is:

$$
X(k)=\left[x(k) v_{x}(k) y(k) v_{y}(k)\right]^{t}
$$




$$
x(k) \text { and } y(k) \text { are } x(k) \text { and } y(k) \text { coordinates of the target. } v_{x}(k) \text { and }
$$

$v_{y}(k)$ are the velocity components of the kth sampling period target. $w\left(k, \Delta t_{x}\right)$ is process noise. $\Delta_{t}$ is the sampling time.

\subsection{Networked multi robot cooperative control system architecture}

The realization of multi-target tracking system based on wireless sensor network provides reliable navigation data for mobile robots. The realization of the multi-target tracking and positioning system brings the wireless sensor network from theory to practice, which lays the foundation for the implementation of the subsequent network feedback control system.

Based on the multi-target tracking and positioning system, the mobile robot serves as a positioning and tracking target. While moving in the grid, the mobile robot can receive the positioning coordinate data fed back by the network, adjust its own movement speed and direction according to different planning tasks, and complete different control tasks.

At present, the platform has been designed and implemented with coordinated control of multiple mobile robots, and can be operated stably. It has been recognized and praised by experts in the same field for many times. According to current understanding, under the support of the National Natural Science Foundation of China, the multitarget tracking and positioning system and its feedback control technology have reached the leading level of domestic counterparts.

The overall system control framework is shown in Figure 3. The networked multimobile robotic cooperative control system is based on the Leader-Follower mode. The information of the Leader and Follower is distributed centrally by the base station through wireless sensor network measurement feedback. The Follower is controlled by the control program shown in Figure 3, and the Leader only needs to follow the local planned trajectory.

Follower local side, control thread and sampling thread were performed at the same time. Multi-thread sampling is to ensure the reliability and timeliness of the sampled data. With the clock of the multimedia high-precision timer control system, the realtime reaction speed of the system is enhanced. In the mobile robot, Kalman filtering is performed on the received network data to reduce the influence of measurement noise, and an effective control strategy and design optimization algorithm are implemented according to planning collaborative tasks. 


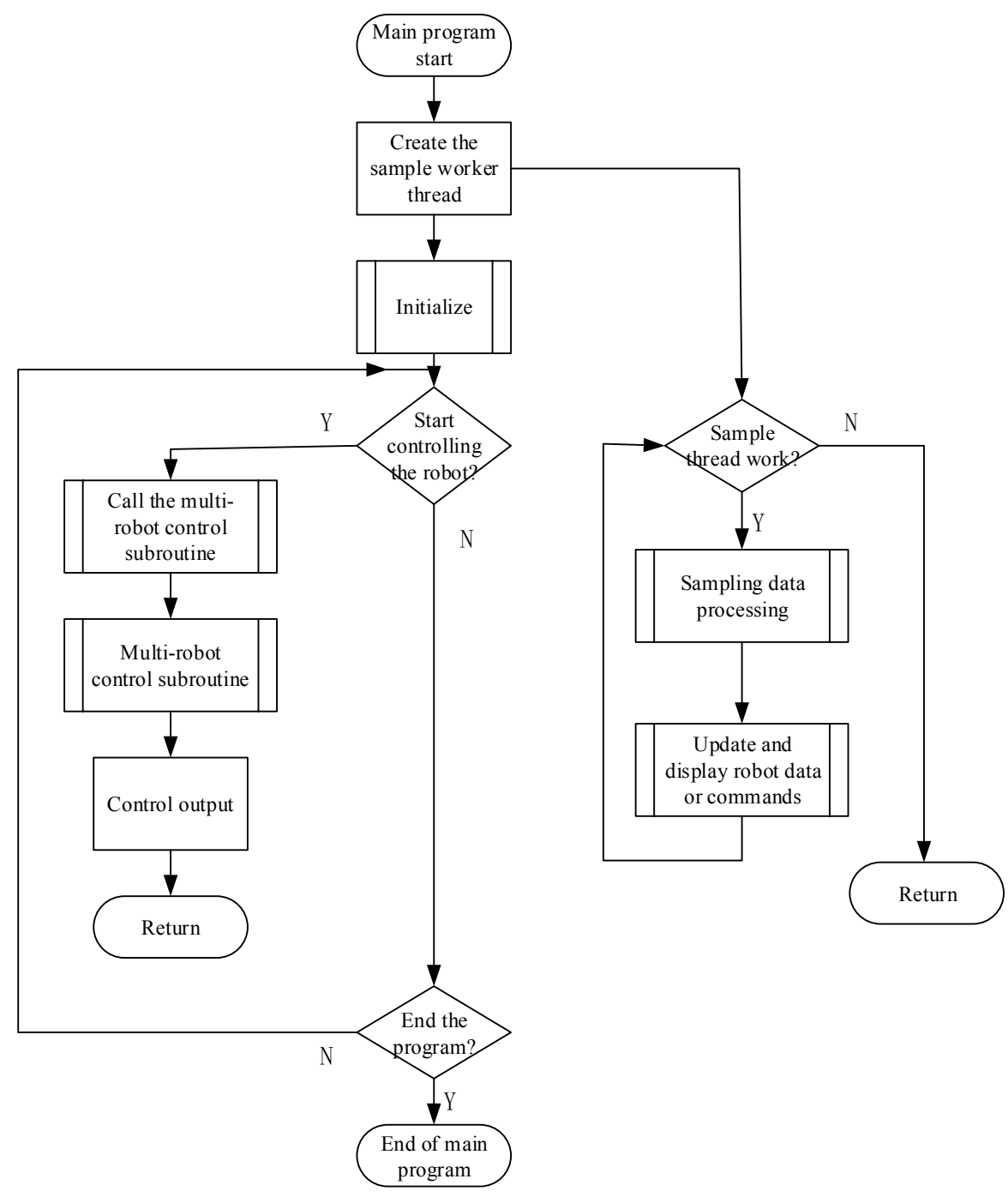

Fig. 3. Networked multi robot cooperative control system architecture

\subsection{Network control system communication protocol and multi-thread sampling}

When a wireless sensor network transmits information to a mobile robot, the communication protocol between the two is defined as follows:

There are two types of information that wireless sensor networks transmit to robots. One is the coordinates $(\mathrm{x}, \mathrm{y})$ of the robot in the wireless sensor network, and the other is the robot motion control command. Currently, there are only two kinds of commands: start or stop. 
The mobile robot uses the Zigbee communication module of the wireless sensor network to communicate with the network. Therefore, the header and tail of the transmitted packet are automatically generated by the Zigbee module. Communication between the communication band and the wireless sensor network node will not conflict. For unified management, the data packets transmitted from the wireless sensor network to the mobile robot include information of the master robot and the slave robot. The robot extracts its own data based on its own status and simultaneously obtains the information of the main robot to track.

According to a predefined communication protocol, the mobile robot parses the received data. The valid data starts from the coordinate/command bit, and respectively marks the transmitted information as coordinate data or control command. The master robot ID number is 01 , the slave robot ID numbers are 02 and 03 respectively, and the robots respectively receive their respective data packets for analysis.

The main controller of the notebook computer receives network data by monitoring the network data transmission serial port converted by the Zigbee communication module. Taking into account the network measurement delay, real-time requirements of the control system and higher actual conditions, the sampling timeliness and accuracy of the network feedback data are the key to the realization of the control system. Therefore, under the WindowsXP system, the most efficient way to obtain serial data based on MFC is sought.

Multithreading is an effective way to increase the real-time processing rate in Windows XP systems. In the XP multitasking system, the CPU time of each process program is determined by the system. There can be multiple concurrent threads in the process to overcome the limitations of the application message queue. Worker threads (as distinguished from user interface threads) are specifically designed to perform background processing tasks such as calculations, scheduling, and serial read/write operations.

In addition, there are three ways to read and write the serial port in MFC: read and write through the MsComm control, read and write through the serial port, and read and write through the Windows API function. From the point of view of program implementation, the MsComm control and the serial port class are all packaged by the Windows API function and provide user interface functions for developers. Thus, its efficiency is lower than reading and writing through Windows API functions.

Therefore, through the Windows API function, it is the most effective way to directly read and write the serial port on Windows platforms such as MFC. Its embodiment is the network sending robot start command. The program implemented by the Windows API function can achieve the effect that the robot responds immediately by clicking a command button, while the other two methods will intermittently "fail". At the same time, it is necessary to use a multi-threading technique to perform sampling separately to make up for the non-real-time bug in the Windows XP system. The sampling period needs to be controlled by a high-precision multimedia timer.

High-precision multimedia timers refer to a set of multimedia timer functions that Microsoft provides to users in the Win32 API to achieve millisecond-level timing. The multimedia clock and system regular timers (SetTimer, regular timer timing interval cannot be less than 55ms) use the same counter work, but the difference is that the 
multimedia timer does not depend on the message mechanism but based on the interrupt. When a certain number of interruptions is reached, the WM_TIMER message in the application message queue is bypassed, and the callback function is directly invoked to respond to the event, thereby ensuring the response speed.

The flow chart of the multi-thread sampling program is shown in Figure 4. The sampling thread and the main thread use the event synchronization method to synchronize the threads. Since variables such as master and slave coordinate information need to be called in both the sampling thread and the main thread, thread synchronization technology is needed to avoid data collisions.

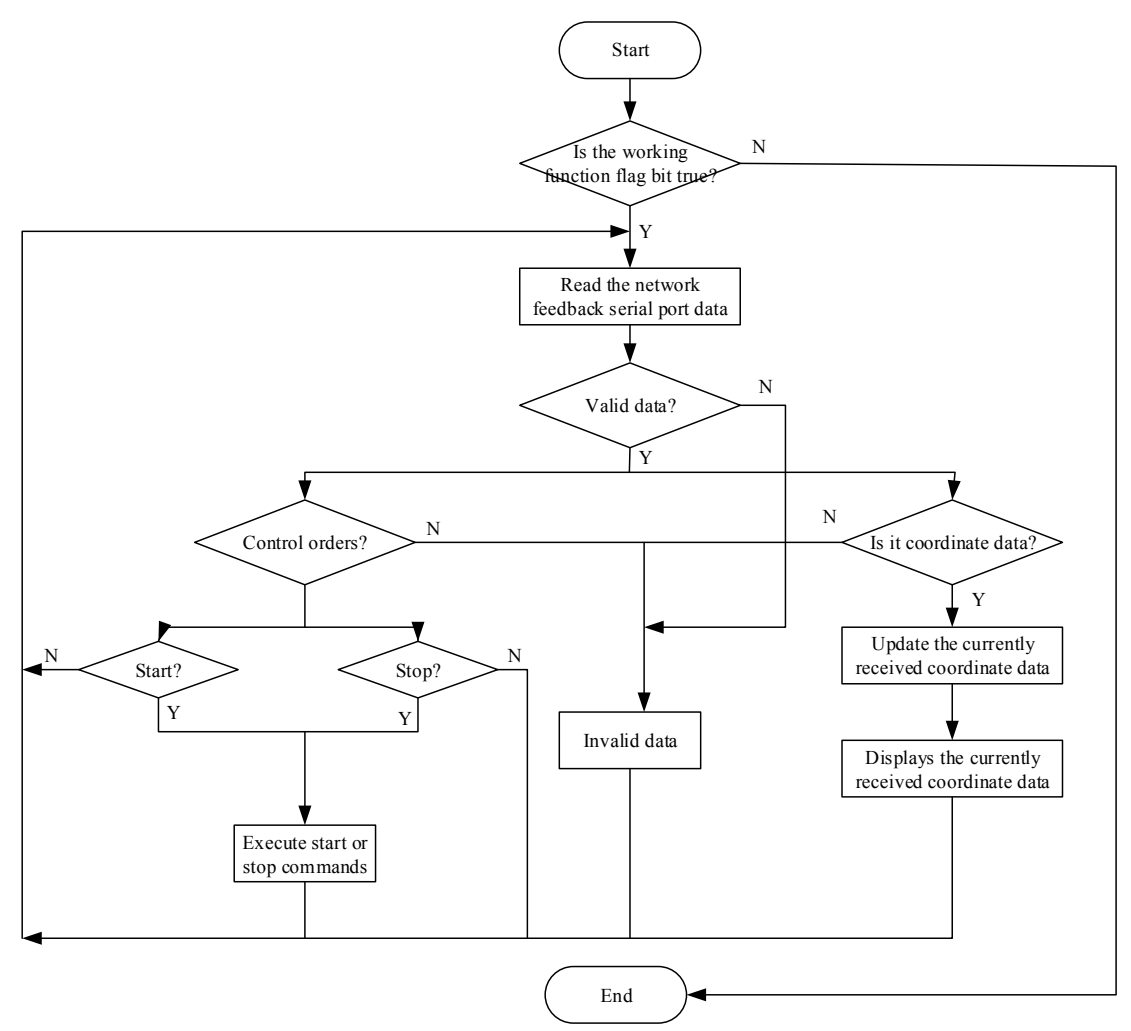

Fig. 4. Flow chart of multi-thread sampling program

To ensure the reliability and timeliness of sampling, the sampling period Ts can be made smaller. Since the laptop is sampled as the main controller, the processor cost can be effectively guaranteed. At the same time, the measurement period of the wireless sensor network needs to be taken into consideration. The sampling period must be much less and fast enough than the measurement period to ensure the success rate of sampling.

In the sampling process, the status of the serial port receiving data is not exactly the same when each Ts arrives. The data in the serial data receive buffer will have different conditions. The positioning data of the next cycle has not yet been sent, and the 
positioning data is being transmitted. Therefore, the reception and parsing of the feedback data from the network must be considered comprehensively so as to get accurate and timely positioning data of the mobile robot. It is prepared for the calculation of the next step of control.

The data packets transmitted to the mobile robot via the wireless sensor network have only two kinds of control commands and coordinate information. Therefore, when the received data packet is a control command, the mobile robot needs to execute a control command immediately. If the coordinate information is received, it is extracted and saved, and the current coordinate data is updated. However, packet loss and timing ambiguity may occur in network transmission. Therefore, if the received data does not conform to the format of the communication protocol, it can be considered as invalid data and discarded. The next sampling cycle is used for sampling.

\section{$4 \quad$ Result analysis and discussion}

\subsection{Numerical simulation and result analysis}

The circular reference track is selected. The reference speed is:

$$
q_{t}=\left[\begin{array}{c}
v_{t} \\
\mathcal{W}_{t}
\end{array}\right]=\left[\begin{array}{l}
0.5 \mathrm{~m} / \mathrm{s} \\
1 \mathrm{rad} / \mathrm{s}
\end{array}\right]
$$

Its radius is $r=0.5 \mathrm{~m}$, and the necessary initial values of the parameters are selected according to the actual physical meaning:

$$
\theta_{t}=\theta_{w}+\frac{\pi}{2}
$$

In the formula, $\theta_{w}$ is the axial angle. $\mathrm{T}$ is the sampling period. The initial pose is:

$$
p_{0}=\left[\begin{array}{c}
0.5 \\
0 \\
\pi
\end{array}\right]
$$

During the simulation experiment, the controller adjustable parameters are $\boldsymbol{k}_{x}, \boldsymbol{k}_{y}$ and $k_{\theta}$. The choice of these three parameters determines the effect of the controller. The specific determination method is as follows:

From the control law and kinematics model, $\boldsymbol{k}_{x}$ directly affects the control speed $\mathrm{v}$, which directly affects the output $\mathrm{x}, \mathrm{y}$. The larger the $k_{x}$, the smaller the steady-state error of $\mathrm{x}$ and $\mathrm{y}$.

From the simulation results, when other parameters are consistent, $k_{x}=\mathbf{2} / \mathbf{s}$ and $k_{x}$ $=\mathbf{5} / \mathbf{s}$ are obtained. 
When $k_{x}=\mathbf{2} / \mathbf{s}, x_{e}(\infty)=0.0042, y_{e}(\infty)=0.0080, \theta_{e}(\infty)=-0.0424$.

When $k_{x}=\mathbf{5} / \mathbf{s}, x_{e}(\infty)=0.0019, y_{e}(\infty)=0.0088, \theta_{e}(\infty)=-0.0472$.

Therefore, $k_{x}$ has a greater impact on $\boldsymbol{X}_{e}$ and $\boldsymbol{\theta}_{e}$. The larger the $\boldsymbol{k}_{x}$, the smaller the steady-state error of x. At the same time, the steady-state error of $\theta_{e}$ is increased.

However, if $\boldsymbol{k}_{x}$ is too large, it can cause oscillations. Under the above parameters, $k_{x}$ cannot exceed 10.1/ $\boldsymbol{k}_{x}$ cannot be equal to or nearly equal to the sampling period $\mathrm{T}$. T is $0.1 \mathrm{~s}$ is $100 \mathrm{~ms}$. When $\boldsymbol{k}_{x}=10 / \mathrm{s}, 1 / \boldsymbol{k}_{x}$ is exactly equal to the sampling period T. Therefore, as the above factors are balanced, the value of $k_{x}$ between 2 and 8 is appropriate.

\subsection{Work results}

In May 2012, the key project of the National Natural Science Foundation of China, "The Theory and Method of Control of Network Systems $(60,774,057)$ " has entered the phase of research and development. As an experimental platform of this project, the real-time tracking and cooperative control system of multiple mobile robots based on wireless sensor network positioning has been basically realized and can operate stably. It includes the $4 \mathrm{~m} \times 6 \mathrm{~m}$ sensor network of 12 wireless sensor network nodes, the realtime tracking and positioning of multi targets, and three mobile robots in the network according to the planning path and the cooperative control of the multi mobile robot.

Wireless sensor networks and mobile robots are integrated. The design and implementation of multi-objective real-time tracking and positioning of wireless sensor network system and networked multi-mobile robot cooperative control system are described. For mobile robots, there are currently three main phases of work:

The first part is the local control of the mobile robot. It includes trajectory tracking, point stabilization, and so on. Through a series of motion characteristics experiments, a specific type of mobile robot control method is mastered, and its development process is designed and a framework for a mobile robot control program is established.

The second part is the design and implementation of multi-mobile robot cooperative control based on wireless sensor networks. The mobile robot is placed in the wireless sensor network, and the navigation information is provided by the sensor network. As the reference input of the mobile robot, the controller is designed to move the mobile robot according to a specified trajectory. There are essential differences between multimobile robot control and stand-alone robot control. The system of multi-target positioning and tracking provides a stable navigation method for cooperative control of multiple mobile robots based on network feedback, and the master and slave robots can maintain synchronization.

The third part is the research of multi-mobile robot cooperative control and its control algorithm. By learning the multi-robot trajectory tracking algorithm, the principles and steps of controller design are mastered. Based on the networked multi-mobile robot cooperative control application in practical projects, the key technologies such as 
collaborative strategy and controller design were deeply studied, and an applicable LQG controller was designed to improve the performance of the control system.

\subsection{Development of project platform}

First, collaborative strategy. The multi-mobile robotic system has advantages that are unmatched by a single mobile robot. It can complete more complex and more difficult tasks. Therefore, the cooperative control system can be used as a multi-robot system control framework. However, as the difficulty of the task increases, more advanced collaborative strategies are needed to complete the task. For example, two mobile robots make a circular nesting movement with a larger radius to form cross links.

Second, the design and implementation of practical controllers. Due to the constraints of such factors as the strong nonlinearity and slip of the mobile robot, the controller's effect in actual control is unsatisfactory. Therefore, a more practical mobile robot model is established. Designing a more effective mobile robot controller will be the top priority in the next phase of the mobile robot control section.

Third, multi-robot formation control. With the stability and scale of the system platform, the disadvantages of large noise at the ultrasonic nodes are becoming increasingly prominent. Therefore, other effective detection methods have also begun to be tested on this platform. Based on this, more and more different robots can move on the platform at the same time. Through multi-sensor information fusion, the problem of measurement conflict caused by multi-robot motion in the grid is resolved. The multi-robot cooperative control at this time will be a more powerful network control system.

In short, with the improvement and development of the system, networked multimobile robot control will be able to achieve more complex and more practical tasks, and the control accuracy will be higher and higher.

\section{Conclusions}

The system of multi-target tracking and tracking provides a stable navigation method for the cooperative control of multiple mobile robots based on network feedback. The mobile robots in the grid are located by a wireless sensor network, and their positioning information is reported to the robots for local control. After obtaining the positioning information from the robots, the robot tracks the trajectory and synchronizes with the main robot according to the mission planning. Various key technologies such as sensor positioning, communication, multi-robot task planning, controller design, and collaborative algorithms are studied and developed. The networked multi-robot cooperative control application mode is explored, which provides a test platform for network control theory and methods. It lays a solid foundation for the design and application of networked multi-mobile robotic cooperative control. According to current understanding, the realization of the platform, technology and corresponding theoretical research have reached the domestic leading level in the field, and even it is a world-class. A networked multi-robot cooperative control system was studied. The key projects of the National Natural Science Foundation were integrated. From the aspects of multi- 
objective real-time tracking and positioning of wireless sensor networks, local control of mobile robots, and networked multi-mobile robotic cooperative control, the realization of a multi-mobile robot coordinated control system based on wireless sensor networks and its controller design are thoroughly described.

\section{$6 \quad$ References}

[1] Palanikumar, R., \& Ramasamy, K. (2018). Effective failure nodes detection using ma-trix calculus algorithm in wireless sensor networks. Cluster Computing, (12): 1-10

[2] Korol, D. R., Spengler, A. W., Gracioli, G., \& Idehara, S. (2018). Low-cost automotive wireless instrumentation: is it possible. Iet Science Measurement \& Technology, 12(1): 3338 https://doi.org/10.1049/iet-smt.2016.0341

[3] Tang, W., Zhang, K., \& Jiang, D. (2018). Physarum-inspired routing protocol for energy harvesting wireless sensor networks. Telecommunication Systems, 67(32): 1-18 https://doi.org/10.1007/s11235-017-0362-8

[4] Szczepny, A., Rogers, S., Wsn, J., Park, K., Mccloy, R. A., \& Cochrane, C. R., et al. (2017). The role of canonical and non-canonical hedgehog signaling in tumor progres-sion in a mouse model of small cell lung cancer. Oncogene, 36(39): 5544-5550 https://doi.org/10.1038/onc.2017.173

[5] Zhu, S., Han, Q. L., \& Zhang, C. (2017). \$1_1\$-stochastic stability and \$1_1\$-gain per-formance of positive markov jump linear systems with time-delays: necessary and sufficient conditions. IEEE Transactions on Automatic Control, 62(7): 3634-3639 https://doi.org/10.1109/TAC.2017.2671035

[6] Oliveira, J. M., Loon, J. T. V., Sloan, G. C., Sewiło, M., Kraemer, K. E., \& Wood, P. R., et al. (2018). Early-stage young stellar objects in the small magellanic cloud. Monthly Notices of the Royal Astronomical Society, 428(4): 3001-3033 https://doi.org/10.1093/mnras/sts250

[7] Berghuis, H., Roebbers, H., \& Nijmeijer, H. (2017). Experimental comparison of pa-rameter estimation methods in adaptive robot control to Automatica, 31(9): 1275-1285 https://doi.org/10.1016/0005-1098(95)00046-Y

[8] Samejima, S., Fozilov, K., \& Sekiyama, K. (2018). Visual support system for remote control by adaptive roi selection of monitoring robot. Robomech Journal, 5(1): 6 https://doi.org/10.1186/s40648-018-0103-0

\section{$7 \quad$ Authors}

Bo Sun works as associate professor at Department of Computer Science \& Technology, Henan Institute of Technology. Xinxiang Henan 453003, China.

Jing-wei Li works as associate professor at Department of Computer Science \& Technology, Henan Institute of Technology. Xinxiang Henan 453003, China.

Guan-ci Yang works as associate professor at Key Laboratory of Advanced Manufacturing Technology of Ministry of Education, Guizhou University, Guiyang, Guizhou, 550003, China.

Article submitted 04 September 2018. Resubmitted 05 October 2018. Fina acceptance 12 October 2018. Final version published as submitted by the authors. 